\title{
Nuclear polarization and entanglement in spin systems
}

\author{
G. B. Furman · V. M. Meerovich • V. L. Sokolovsky
}

Published online: 13 March 2009

(C) Springer Science+Business Media, LLC 2009

\section{Erratum to: Quantum Inf Process DOI 10.1007/s11128-009-0103-3}

There are errors in Figs. 2 and 3 and their captions. The corrected figures and captions follow.

The online version of the original article can be found under doi:10.1007/s11128-009-0103-3.

G. B. Furman $(\bowtie)$ · V. M. Meerovich · V. L. Sokolovsky

Department of Physics, Ben-Gurion University of the Negev, P.O. Box 653, Beer-Sheva 84105, Israel e-mail: gregoryf@bgu.ac.il

G. B. Furman

Ohalo College, P.O. Box 222, Qazrin 12900, Israel 

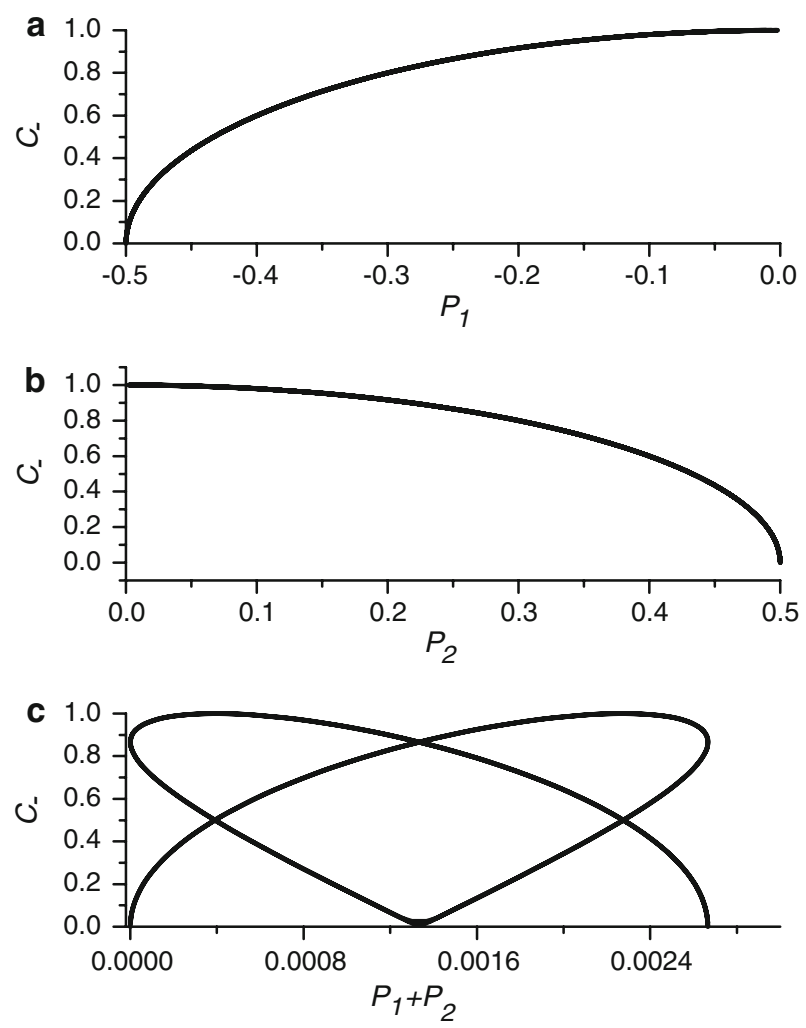

Fig. 2 Concurrence as a function of the polarization for antiferromagnetic initial state (6): a polarization of the first spin, $P_{1} ; \mathbf{b}$ polarization of the second spin, $P_{2} ; \mathbf{c}$ the total spin polarization, $P_{1}+P_{2}$ 

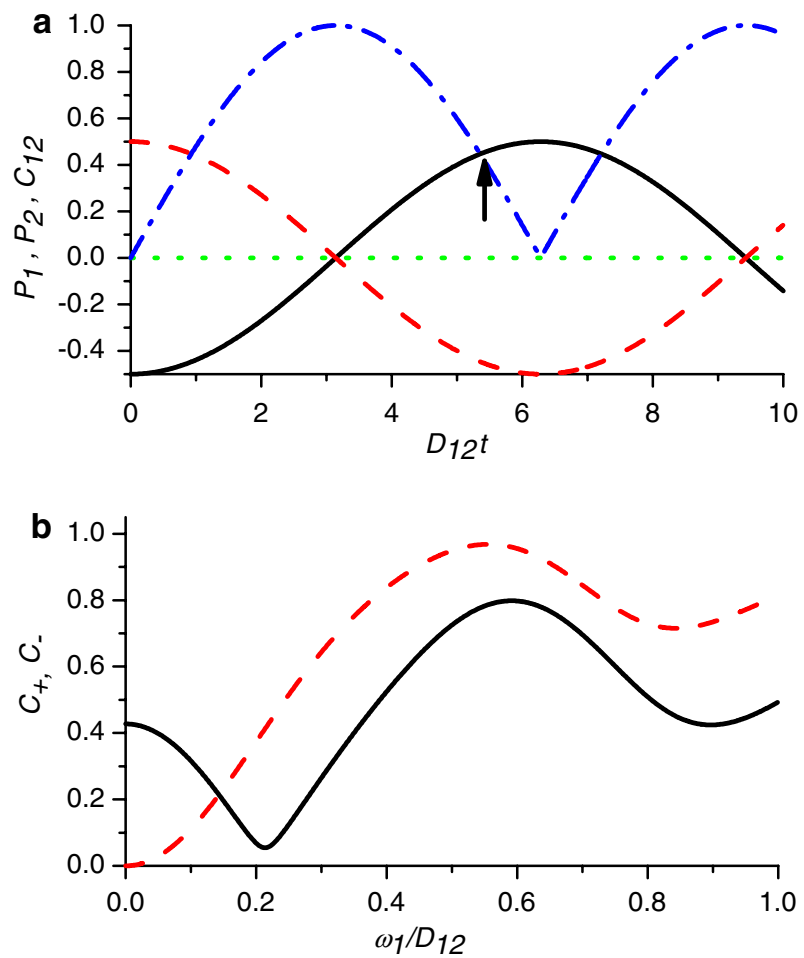

Fig. 3 a Dynamics of the polarization and concurrence without an irradiation field (at $\left.\omega_{1}=0\right)$ for the initial condition (4). Black dashed curve: polarization $P_{1-}(t)$ of the first spin. Red dashed curve: polarization $P_{2-}(t)$ of the second spin. Green dotted curve: total polarization $P$. Blue solid curve: concurrence $C_{-}(t)$. The arrow marks the point $D_{12} t=5.4 \mathbf{b}$ Concurrence as a function of the irradiation field strength $\omega_{1} / D_{12}$ at time $D_{12} t=5.4$ for various initial conditions. Black solid curve: $C_{-}$for the initial condition (4). Red dashed curve: $C_{+}$for the initial condition (5) (Color figure online) 